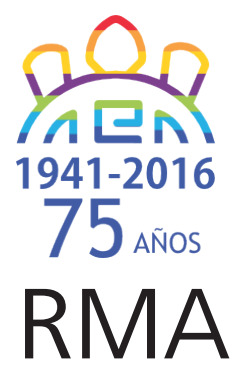

Arqueología

\title{
Análisis tecno-morfológico, morfométrico y de materias primas de los esferoides líticos de la costa del Golfo San Matías (Río Negro, Argentina)
}

\author{
Techno-morphological, morphometrics and raw \\ materials analyses of lithic spheroids from San Matías \\ Gulf (Rio Negro, Argentina)
}

Jimena Alberti* y Marcelo Cardillo**

*Instituto Multidisciplinario de Historia y Ciencias Humanas (IMHICIHU)-CONICET. E-mail: jimealberti@gmail.com. **Instituto Multidisciplinario de Historia y Ciencias Humanas (IMHICIHU)-CONICET.

E-mail: marcelo.cardillo@gmail.com

\begin{abstract}
Resumen
En este trabajo se presenta una caracterización inicial de la muestra de bolas líticas recuperadas en la costa rionegrina del golfo San Matías, en sitios fechados en el Holoceno medio y tardío. Con este fin se aplicaron distintos métodos de análisis métrico, morfométrico (análisis de forma) y de materias primas, así como de caracterización tecnomorfológica de los artefactos. Los resultados indican la existencia de dos grandes grupos de bolas: uno conformado por artefactos de tamaño mediano-pequeño de contorno irregular, tendientes a una forma cuadrangular y con una vista lateral plana; y otro de artefactos de forma más esférica y de tamaño mayor. Asimismo fue posible constatar un uso predominantemente local de rocas para la manufactura de estos instrumentos, lo cual habría incidido en las diferencias de peso que fueron detectadas entre los instrumentos recuperados en ambas porciones de la costa rionegrina (norte y oeste). Por último, la presencia de marcas de formatización en la mayor parte de la muestra y la baja presencia de piezas con pulido en toda su superficie indicarían una baja inversión de energía puesta en la manufactura de los instrumentos.
\end{abstract}

Palabras clave: bolas líticas; golfo San Matías; análisis morfométricos; materias primas.

\begin{abstract}
In this paper we present an initial characterization of the sample of bolas recovered in archaeological sites located at San Matías Gulf coast (Río Negro province) dated in the Middle and Late Holocene. For this purpose different methods of metric, morphometric (shape analysis) and raw materials analyses, as well as a techno-morphological characterization of the artifacts, were applied. The results indicate the existence of two large groups of bolas: one is integrated by artifacts of medium-small size and irregular outline, which tend to a quadrangular shape with a flat side view; and other group of artifacts which are larger and with a more spherical shape. It was also possible to establish the use of mainly local raw materials for the manufacture of these instruments, which would have affected the weight differences we detected between the bolas recovered in both portions of the coast (North and West). Finally, the presence of formatization traces in most of the sample and the low presence of pieces with polish over their entire surfaces, would indicate a low investment of energy put into the final manufacture of these artifacts.
\end{abstract}

Keywords: bolas; San Matías Gulf; morphometrics analyses; lithic raw materials.

Los esferoides líticos han sido utilizados en PampaPatagonia como armas de caza o para la guerra. En Patagonia en particular, los hallazgos de esta tecnología se encuentran ampliamente distribuidos desde el Holoceno temprano (ver Borrazzo y Etchichury 2013 y citas alli) hasta alrededor de los 1000 años AP. Sin embargo, los análisis de estos artefactos han sido escasos hasta el momento. Entre algunos autores que abordaron su estudio en la mencionada región se cuentan los trabajos de González (1954), Mansur-Franchomme et al. (1987-1988), Torres (2009), Torres y Morello (2011), Borrazzo y Etchichury (2013), entre otros. De acuerdo con Oswalt (1976), las esferas líticas son consideradas armas utilizadas para matar o lesionar. Las llamadas "boleadoras" comprenden diferentes tipos de armas, dependiendo del tipo de presa a capturar y del número de ramales y el peso y tamaño 


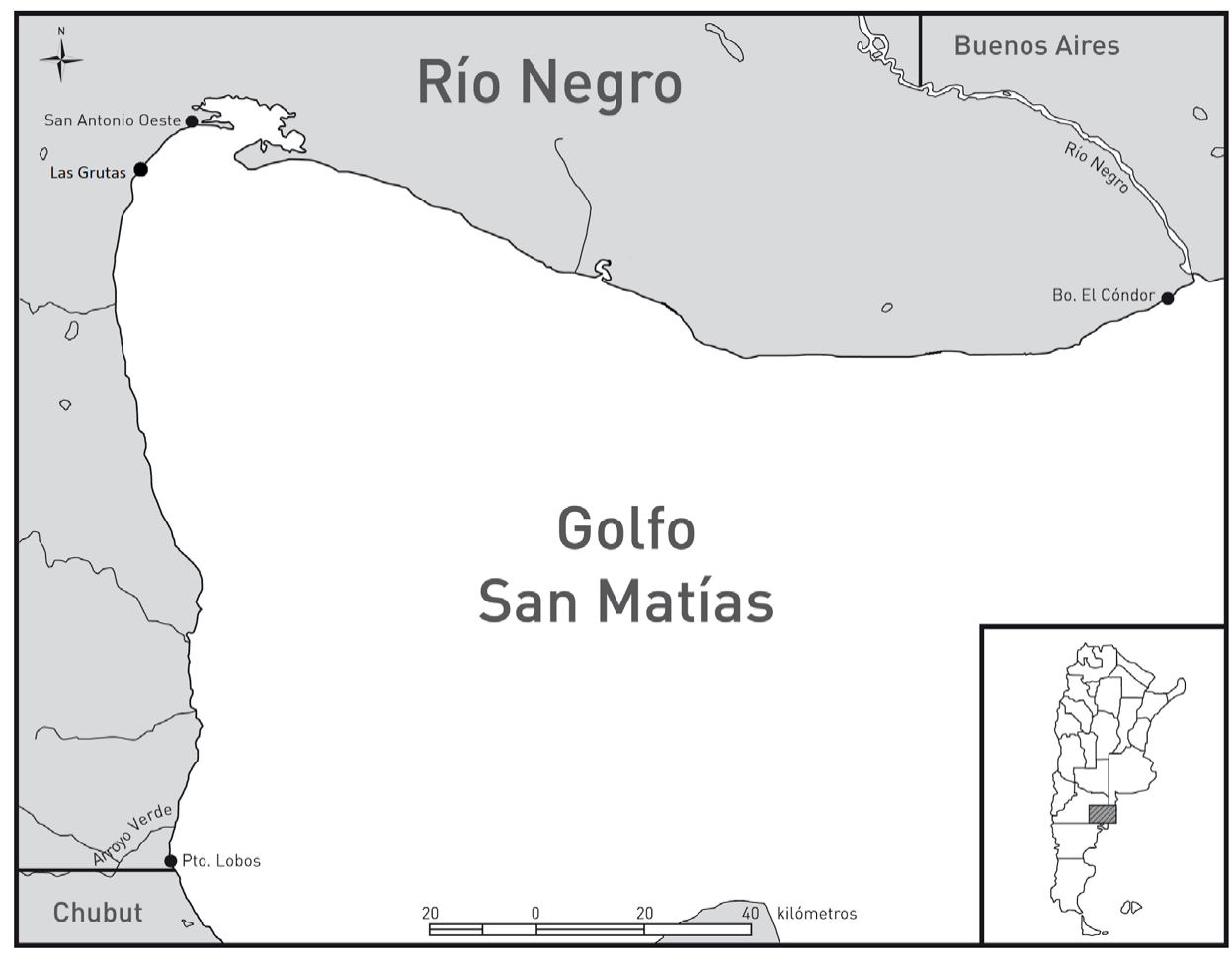

Figura 1. Localización del área de estudio. Se señalan los puntos que delimitan ambas porciones del golfo.

Figure 1. Localization of the study area. The points used to delimitate both portions of the Gulf are marked.

de las bolas en sí (entre otros, Bird 1993; Martinic 1995; Vecchi 2007). Teniendo en cuenta estas variables, existen diferentes tipos de boleadoras: las boleadoras de dos y tres ramales, utilizadas para la caza del ñandú y el guanaco respectivamente, y la llamada "bola perdida" de un solo ramal y que se habría utilizado en los enfrentamientos armados (González 1954; Bird 1993; Martinic 1995; Vecchi 2007). Sin embargo, también se ha reportado el uso de esta arma para cazar animales de diferentes portes, como zorros, mulitas, aves, lobos marinos, pumas, venados e incluso vacas y caballos (Musters 1964). Para nuestra área de estudio, nos interesa en particular destacar la propuesta de Moreno (2008), quien postula el uso de estas esferas líticas como "rompecráneos", utilizados para la caza de lobos marinos. Moreno propone esta posible funcionalidad para artefactos subesféricos o irregulares de gran tamaño y peso que poseen un semi-surco o surco incompleto y que en general, se han encontrado en la costa de Chubut asociados a restos de lobos marinos (Moreno et al. 2000). En este sentido, estos animales habrían sido capturados mediante un lazo cuando se hallaban en la costa y rematados con estas mazas líticas (Sánchez Labrador 1936 en Vecchi 2007). Cabe destacar, además, que estos instrumentos también pueden haber sido utilizados en el pasado para la lucha o defensa (Gonzalez 1954) o como pesas de red, tal como ha sido mencionado para el área en trabajos previos (Scartascini 2014).

En este trabajo se presenta por primera vez el análisis de un conjunto de esferas y esferoides líticos recuperado en la costa del golfo San Matías, en la provincia de Río Negro (Argentina). Para ello se determinaron las materias primas líticas sobre las que las mismas fueron confeccionadas y se realizaron análisis tecno-morfológicos y de morfometría geométrica para establecer la variabilidad de formas y tipos dentro de los conjuntos recuperados. No buscamos determinar la funcionalidad de estos instrumentos sino sistematizar por primera vez la información sobre las esferas líticas recuperadas en el área de estudio.

\section{El área de estudio}

La costa del golfo San Matías (provincia de Río Negro, Argentina) (Figura 1) es una cuenca alargada en sentido $\mathrm{N}-\mathrm{S}$, con una longitud de aproximadamente $380 \mathrm{~km}$ lineales, entre Puerto Lobos (en el límite con la provincia de Chubut) y el balneario El Cóndor, en la desembocadura del río Negro en el océano Atlántico. Debido a que esta área presenta características geológicas y geomorfológicas que no son constantes a lo largo del espacio, ha sido dividida en dos sub-sectores para su estudio: la costa norte, entre la ciudad de San Antonio Oeste y El Cóndor, y la costa oeste, entre la ciudad de Las Grutas y el límite con Chubut. La costa norte se caracteriza por su gran bioproductividad, dada por la combinación particular de la presencia de agua dulce en dunas, la existencia de reparos topográficos, el acceso a especies marinas (moluscos, peces y lobos marinos) y la disponibilidad de rocas aptas para la talla (Borella 2006; Favier Dubois y Borella 2011). Corre de oeste a este y presenta diferentes rasgos, entre los que cabe señalar planicies elevadas de rodados, cordones medanosos, bajos y una zona litoral, en la que se alternan playas con fácil acceso al mar con acantilados abruptos (González Díaz y Malagnino 1984). Las fechas de los sitios localizados en el área se ubican entre los 6000 y los 450 años ${ }^{14} \mathrm{C}$ AP (Favier Dubois y Borella 2011).

Por su parte, la costa oeste presenta una disponibilidad 
de recursos menor, escasez de agua dulce y de reparos topográficos. A diferencia de lo que sucede en la costa norte, esta área corre de norte a sur y sus principales rasgos geomorfológicos están constituidos por acantilados y serranías. Los fechados de las localidades arqueológicas de esta área se ubican entre los ca. 3200 y los 700 años 14C AP (Borella et al. 2015).

En la costa rionegrina del golfo han sido ubicados más de 60 loci, tanto concheros como concentraciones de materiales en la superficie de las terrazas y en las hoyadas de deflación entre médanos, agrupados en diversas localidades (Favier Dubois et al. 2008; Favier Dubois y Borella 2011). Los concheros, en general de escasa potencia (Favier Dubois y Borella 2011), están formados principalmente por valvas de mejillones y cholgas, muchas veces quemadas y con carbones entre ellas. En estos loci se han recuperado tiestos, artefactos líticos, óseos y sobre valva, cáscaras de rheidos grabadas, material arqueofaunístico, y enterratorios humanos, entre otros.

En relación con las materias primas líticas aptas para la talla, en la costa norte del golfo se han localizado únicamente fuentes secundarias, abundantes y ubicuas en el ambiente, compuestas por rodados de tamaños variables, de rocas principalmente volcánicas, de calidad variable para la talla (Alberti 2015; Alberti et al. 2015; Favier Dubois y Alberti 2014). Por otro lado, en la costa oeste las fuentes secundarias son más escasas, se encuentran localizadas en lugares puntuales del espacio y presentan rocas de variadas litologías, de calidad buena a excelente para la talla (Alberti 2015; Alberti y Cardillo 2015; Alberti et al. 2015). En esta última porción de la costa del golfo se han localizado cuatro fuentes primarias: dos de sílice, una de toba y una de rocas metamórficas de calidad mala para la talla. Una de las fuentes de sílice y la de toba presentan evidencias claras de explotación (Alberti 2015; Alberti y Cardillo 2015; Alberti et al. 2015).

\section{Materiales y metodología de análisis}

Las denominadas bolas líticas se agrupan dentro de los materiales líticos manufacturados mediante técnicas de picado y/o abradido (incluyendo el alisado, el pulido y/o el bruñido) (Babot 2004). En este trabajo se analizaron 42 artefactos recuperados en siete localidades arqueológicas de las costas norte y oeste del golfo San Matías en contextos de superficie. El análisis de las materias primas sobre las que fueron manufacturados estos instrumentos se realizó según los criterios establecidos en Alberti y Fernández (2015), mientras que para el análisis tecnotipológico se siguieron los lineamientos de Vecchi (2010). Para este último análisis se consignó para cada pieza:

1) medidas: tomadas con calibre digital y expresadas en milímetros. En caso de las piezas sin surco, el diámetro 1 es el correspondiente al eje mayor de la pieza. En el caso de las bolas con surco, el diámetro 1 es el paralelo al mismo;
2) peso: expresado en gramos, tomado con balanza digital;

3) morfología: en este caso se siguió a González (1954); por ejemplo, sólidos de revolución, con protuberancias, esféricas, entre otras.

4) estado del artefacto: entero, fragmentado o fragmento: por artefacto fragmentado entendemos a aquellas piezas que presentan más del $51 \%$ de su volumen, mientras que aquéllas de las que se recuperó un porcentaje menor son consideradas fragmentos;

5) acabado de la superficie: grado 0 (superficie sin pulido, solamente picada), grado 1 (pieza con alisado inicial o pulido irregularmente distribuido en su superficie), grado 2 (pulido intenso que cubre totalmente la pieza).

6) surco: consignado como presencia/ausencia. En caso de estar presente, se describió ancho en su parte externa, profundidad en su parte interna, técnica de elaboración (picado o pulido) y estado de elaboración (completo o incompleto);

7) marcas de formatización: por ejemplo, aristas o negativos de lascados sin eliminar;

8) alteraciones postdepositacionales: por ejemplo, marcas de raíces, presencia de costras de diferentes tipos.

9) evidencias de reciclaje: bordes de fractura regularizados, sectores con pulimento intenso, entre otras;

10) evidencias de descarte: fragmentos con estrías, saltaduras, marcas de percusión, etc.;

11) peso (en gramos) y peso específico de las materias primas (peso/volumen).

12) la morfología, determinada partir de imágenes digitalizadas, las cuales fueron empleadas para análisis de Fourier elíptico (ver más adelante).

\section{Análisis estadísticos}

Variables cuantitativas

Los análisis de forma fueron realizados sobre las piezas enteras o aquéllas cuyas fracturas no impidieran el registro de las variables métricas o morfológicas $(n=20)$. Para obtener información de los contornos, se empleó el análisis de Fourier Elíptico (EFA) (Kuhl y Giardina 1982; Rohlf 2006). Este método permite una descripción cuantitativa de la forma, la cual puede ser empleada para describir o comparar conjuntos de objetos a partir de sus contornos. El análisis de Fourier elíptico se aplica en contornos cerrados, donde la descripción de cada uno se realiza a partir de $n$ parámetros denominados armónicas, cada una de las cuales contribuye a la descripción de la forma empírica en diferente grado. Las primeras armónicas describen un contorno de una manera muy general (variación de escala amplia, tendencias generales), mientras que las últimas describen variaciones que ocurren a pequeña escala (variaciones locales, casos particulares), de forma tal que descomponen todas las posibles dimensiones de la morfología. Variaciones globales pueden ser el redondeamiento, alargamiento, compresión, mientras que las más locales involucran comúnmente la angularidad, aristas, depresiones, etc. Por este motivo, es frecuente que la variación descripta por las 
últimas armónicas contenga información menos relevante y sea dejada de lado. Posteriormente, las armónicas son normalizadas para eliminar el efecto del tamaño, orientación y rotación del objeto digitalizado. Tal como lo mencionamos, la utilización de esta metodología tiene como principal ventaja realizar una descripción cuantitativa de la forma de manera continua. Esto disminuye, entre otras cosas, la subjetividad de la definición tipológica o en categorías de forma cuya variación se comporta muchas veces como un continuo (Gero y Mazzullo 1984), además de permitir su tratamiento estadístico.

Para la digitalización de los contornos se emplearon fotografías digitales de las vistas frontal y lateral, registradas a ocho megapíxeles. Dado que en su mayoría se trata de formas subesféricas, con una cara más redondeada o expandida y otra relativamente más plana o comprimida, se denominó frontal a la primera y lateral a la segunda. En el caso de las morfologías casi perfectamente esféricas (en las que resultó imposible utilizar este criterio de forma coherente), la vista "frontal" se seleccionó al azar y luego se orientó la pieza en dirección ortogonal para la determinación de la vista lateral. En cada contorno se distribuyeron 100 puntos equidistantes a partir del programa TPSdig2 (Rohlf 2006), que fueron la base para la estimación de las armónicas. Los archivos de puntos fueron procesados mediante el programa Momocs 1.0.0 (Bonhomme et al. 2014) de R (R Development Core Team 2009) en donde se realizó, previamente, la estandarización de los contornos en base a la primer armónica. Esta estandarización remueve la información relativa al tamaño, posición y rotación de cada ejemplar, de manera tal que se preserva sólo aquella relativa a la forma. La cantidad de armónicas mínimas necesarias para describir correctamente el contorno fue determinada visualmente. Reducir el número de estos parámetros permite tomar el número mínimo de descriptores y dejar de lado pequeñas variaciones relativas a errores aleatorios producidos durante la copia o digitalización de los contornos.

Debido a que la matriz de armónicas es muy extensa en relación a la cantidad de casos, es un procedimiento habitual realizar un análisis de componentes principales con el fin de obtener las tendencias generales de variación morfológica en la muestra. Esto permite obtener las tendencias principales de variación morfológica y dejar de lado patrones de escala muy pequeña, comúnmente vinculados a factores aleatorios, como irregularidades del contorno o la influencia de casos atípicos. Las nuevas variables resultantes del análisis de componentes principales fueron empleadas en análisis descriptivos y comparativos junto con las demás variables categóricas y continuas con el fin de explorar la relación entre aspectos tecnológicos y morfológicos.

Posteriormente, con el fin de explorar patrones de agrupamiento morfológicos, los tres primeros componentes principales de cada vista, que suman al menos $90 \%$ de la variabilidad morfológica total de cada sección, fueron empleados en análisis de agrupamiento jerárquico utilizando el método de varianza mínima de Ward (Legendre y Legendre 2012). El empleo de métodos de agrupamiento sobre componentes principales o sobre variables resultantes de otros métodos multivariados mejora las posibilidades de clasificación y/o reconstrucción de patrones debido a que los componentes principales han previamente particionado la varianza original, separando el componente aleatorio que tiende a formar parte de los últimos componentes (Husson et al. 2010). El método de agrupamiento usado por el método de Ward es el de unir pares de casos tal que aumenten lo menos posible la inercia dentro de cada grupo en cada paso de un proceso de clasificación jerárquica. El resultado es el de maximizar la diferencia entre grupos y minimizar la varianza hacia su interior con el mínimo posible de particiones en un dendograma o árbol de disimilitud creciente (Kauffman y Rosseeuw 1990).

Para determinar la vinculación entre forma y tamaño, se realizó la correlación entre los tres primeros componentes principales de forma para cada una de las vistas (frontal y lateral) y tres variables métricas (volumen, peso total y peso específico o peso de la materia prima, estimado realizando peso/volumen). Un aspecto importante al comparar forma y tamaño, es que éste último depende en gran medida del tipo de roca utilizado, ya que para la manufactura de los instrumentos analizados se emplearon variedad de materias primas (Alberti 2015), algunas de ellas de gran densidad, como las rocas plutónicas. Así, artefactos del mismo tamaño no son directamente comparables porque pueden poseer un peso muy diferente, siendo ésta una variable de gran importancia en este tipo de instrumentos. La determinación del peso específico a partir del cociente de peso/volumen permite explorar la vinculación entre el tipo de materia prima y morfología, tamaño e historia de vida de los artefactos.

Por último se evaluaron las diferencias entre las variables cuantitativas antes mencionadas y la procedencia y materia prima utilizada en su confección. Las diferencias entre sectores fueron evaluadas para estas tres variables en base a los valores medios mediante el test de la $t$ en caso de distribuciones de igual varianza, y mediante el test de la t para varianzas desiguales (o test de Welch) y permutaciones sobre la distribución de t en el caso de que el requisito de heterocedasticidad no fuese cumplido. En el caso de la morfología, las diferencias entre sectores fueron contrastadas empleando el $90 \%$ de la variación explicada por los componentes principales para cada vista a través de análisis discriminante y tomando la procedencia (costa norte u oeste) como factor. El análisis discriminante busca el eje de máxima separación entre los conjuntos de datos en función de los factores determinados a priori. La significancia de esta separación fue determinada mediante el análogo multivariado al test 
Tabla 1. Distribución de los ejemplares estudiados por estado, tipo y subtipo y materia prima. A: corresponde a las bolas sin surco. A.a: esférica. A.b: sólido de revolución. A.c.1: piezas sin surco con forma de dos troncos de cono unidos por su base. B: corresponde a las bolas con surco. B.a: esférica. B.b: sólido de revolución. B.c.1: sólido de revolución con surco perpendicular al eje mayor. D: con protuberancias múltiples.

Table 1. Distribution of artifacts studied by condition, type and subtype and raw materials. A: corresponds to bolas without groove. A.A: spherical. A.b: revolution solid. A.c. 1: pieces with not groove shaped as two truncated cones joined by their base. B: it bolas with groove. B.A: spherical. B.b: revolution solid. B.c.1: revolution solid of with groove perpendicular to the major axis. D: with multiple protuberances.

de la t o test de Hotelling $\mathrm{t}^{2}$ (Davis 1986).

\section{Variables cualitativas}

Las diferencias entre variables categóricas vinculadas a localización de las muestras, acabado, materia prima y presencia-ausencia de surco fueron testeadas mediante el test de independencia de $\mathrm{Chi}^{2}$ comúnmente utilizado para la comparación entre variables categóricas. Debido a las diferencias en las materias primas empleadas en cada sector de la costa, el tamaño de las muestras entre y dentro de cada nivel de las variables fluctúa, siendo en algunos casos, cercano o igual a 0 . Por este motivo se empleó el procedimiento de Monte Carlo para la estimación de los valores de probabilidad ya que este último posee mejor performance en este tipo de situaciones (Hope 1968). Asimismo, se estimó la potencia del test (Cohen 1988) para detectar diferencias entre las muestras. La potencia $(P)$ depende, entre otras cosas, del tamaño de la muestra y del efecto tamaño. Este último puede entenderse como el grado de diferencia entre dos muestras. Cuanto mayor es el tamaño de la muestra más potencia posee el test para detectar diferencias cada vez más pequeñas. Por otro lado, test basados en muestras pequeñas requieren que el efecto sea más grande para poder detectarlo (un efecto de 0.5 o más según Cohen 1988). En este trabajo, consideramos este nivel efecto para evaluar la potencia de los test realizados. Asimismo, tomamos un nivel de potencia cercano a 0.8 como un valor confiable para rechazar convenientemente $\mathrm{la}_{0}$ de no diferencia. El valor de potencia se presenta en los casos en que el resultado es significativo.

Los análisis estadísticos se realizaron mediante el programa R 3.1.3 (R Development Core Team 2009) para los análisis morfométricos y el test de potencia, y el Past 2.17c (Hammer et al. 2001) para los test de hipótesis.

\begin{tabular}{|c|c|c|c|c|}
\hline Caso & Área & Estado & Tipo y subtipo & Materia prima \\
\hline 1 & $\mathrm{~N}$ & Entero & A.a & Sedimentita \\
\hline 2 & N & Entero & A.b & Volcanita básica \\
\hline 3 & $N$ & Entero & B.b & Volcanita básica \\
\hline 4 & $\mathrm{~N}$ & Fragmentado & $A$ & Volcanita ácida \\
\hline 5 & N & Fragmentado & D & Volcanita ácida \\
\hline 6 & $\mathrm{~N}$ & Fragmentado & D & Volcanita ácida \\
\hline 7 & N & Fragmento & A & Plutonita ácida \\
\hline 8 & $\mathrm{~N}$ & Entero & B.b & Volcanita ácida \\
\hline 9 & $N$ & Fragmentado & B.c. 1 & Caliza \\
\hline 10 & $N$ & Fragmentado & B.b & Caliza \\
\hline 11 & N & Entero & A.b & Volcanita básica \\
\hline 12 & $N$ & Fragmento & A & Caliza \\
\hline 13 & $N$ & Fragmentado & $A$ & Caliza \\
\hline 14 & N & Fragmento & $A$ & Caliza \\
\hline 15 & N & Fragmento & B & Caliza \\
\hline 16 & $\mathrm{~N}$ & Fragmentado & B & Caliza \\
\hline 17 & $\mathrm{~N}$ & Fragmentado & B.b & Sedimentita \\
\hline 18 & $\mathrm{~N}$ & Fragmento & B & Caliza \\
\hline 19 & $\mathrm{~N}$ & Fragmento & B & Caliza \\
\hline 20 & $\mathrm{~N}$ & Fragmentado & B & Caliza \\
\hline 21 & N & Entero & B.b & Caliza \\
\hline 22 & $\mathrm{~N}$ & Entero & B.a & Caliza \\
\hline 23 & $\mathrm{~N}$ & Entero & B.b & Caliza \\
\hline 24 & N & Entero & B.c.1 & Indeterminada \\
\hline 25 & $\mathrm{O}$ & Fragmento & Indeterminado & Volcanita \\
\hline 26 & 0 & Fragmentado & A & Hematita \\
\hline 27 & $\mathrm{O}$ & Fragmentado & B.b & Volcanita \\
\hline 28 & 0 & Entero & A.c. 1 & Volcanita \\
\hline 29 & $\mathrm{O}$ & Entero & A.b & Volcanita \\
\hline 30 & $\mathrm{O}$ & Fragmento & Indeterminado & Hematita \\
\hline 31 & $\mathrm{O}$ & Fragmento & $A$ & Volcanita ácida \\
\hline 32 & $\mathrm{O}$ & Entero & A.b & Hematita \\
\hline 33 & $\mathrm{O}$ & Entero & A.b & Hematita \\
\hline 34 & $\mathrm{O}$ & Entero & A.b & Hematita \\
\hline 35 & $\mathrm{O}$ & Entero & A.a & Hematita \\
\hline 36 & $\mathrm{O}$ & Entero & A.a & Hematita \\
\hline 37 & $\mathrm{O}$ & Entero & A.a & Hematita \\
\hline 38 & $\mathrm{O}$ & Fragmento & Indeterminado & Hematita \\
\hline 39 & 0 & Entero & A.a & Diorita \\
\hline 40 & $\mathrm{O}$ & Entero & B.c.1 & Hematita \\
\hline 41 & $\mathrm{O}$ & Entero & ¿Rompecráneos? & Hematita \\
\hline 42 & $\mathrm{O}$ & Entero & A.a & Hematita \\
\hline
\end{tabular}

\section{Resultados}

La muestra analizada comprende un total de 24 bolas provenientes de la costa norte y 18 de la costa oeste (ver algunos ejemplos en la Figura 2). Cabe recordar que para los análisis morfométricos y estadísticos fueron empleados únicamente aquellos ejemplares que se encontraban enteros, excepto en el caso de los test de $\mathrm{Chi}^{2}$. La distribución de tipos morfológicos del conjunto se muestra en la Tabla 1:

Tal como se desprende de la tabla, el 50\% de la muestra corresponde a ejemplares enteros, el $23.8 \%$ a ejemplares fragmentados y el restante $26.2 \%$ a fragmentos de 


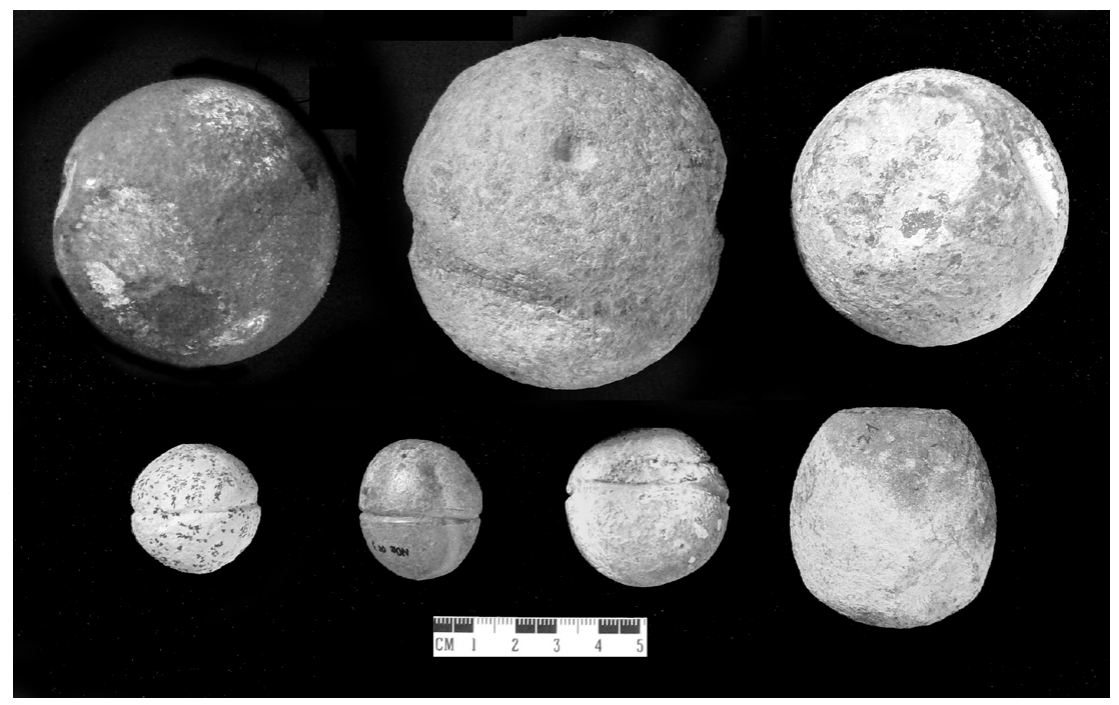

Figura 2. Esferas líticas recuperadas en la costa del golfo San Matías. En la línea superior de la figura se observan dos esferas líticas sin surco en los extremos, mientras que en la línea inferior hay piezas con surco correspondientes a sólidos de revolución. En el extremo inferior derecho se observa una bola sin surco con uno de sus extremos truncos.

Figure 2. Bolas recovered in San Matías Gulf coast. In the top line of the figure, there are two lithic pieces without groove, while in the bottom line there are bolas with groove corresponding to solids of revolution. In the bottom right, a ball without groove with one truncated end is observed.

bolas. Predominan aquellas bolas sin presencia de surco (47.6\% del total) por sobre las que sí lo poseen (38.1\%), mientras que en tres de los ejemplares no fue posible determinar el tipo y subtipo debido a que estaban muy fragmentados. Finalmente se registró la presencia de un posible rompecráneos (en el sentido de Moreno 2008 y Moreno et al. 2000) y de dos ejemplares de bolas con protuberancias, aunque fragmentadas. En relación con las materias primas, se realizaron cortes delgados sobre cuatro ejemplares que sirvieron luego de referencia para la identificación macroscópica del resto de las rocas (Alberti 2015). El 30.9\% de la muestra corresponde a bolas manufacturadas sobre caliza, el $28.6 \%$ a ejemplares de hematita, un $28.6 \%$ a rocas volcánicas en general, el $4.8 \%$ a rocas sedimentarias no identificadas, el $2.4 \%$ a un ejemplar de diorita, $2.4 \%$ a una roca plutónica ácida, y el $2.4 \%$ restante a una roca. Todas las materias primas son de origen local (Alberti 2015) y pueden ser halladas en las fuentes primarias y/o secundarias del área de estudio.

En relación con las marcas de formatización, en un $59.6 \%$ (25 piezas) se registraron negativos de lascado y un $7.1 \%$ de los casos presentaba aristas sin rebajar (3 casos), mientras que el restante 30.9\% (13 bolas) no poseía marcas de formatización. Respecto del acabado de las piezas, 15 piezas (35.7\%) tienen acabado 0 (superficie sin pulir), 21 piezas (50\%) acabado 1 (alisado inicial o pulido irregularmente distribuido) y las seis piezas restantes (14.3\%) acabado 2 (pulido intenso en toda la pieza). Finalmente cabe destacar que no hemos registrado evidencias de reciclaje y que el descarte de estos artefactos puede haber sido por fractura durante el uso o la manufactura, o se pudieron haber descartado cuando aún su formatización no estaba finalizada.

El promedio de peso para toda la muestra analizada es de 355 g, un desvío estándar de 205 g con un rango amplio de pesos, que oscila entre 28 y 628 g (Figura 3). Esta amplitud está vinculada a las diferentes materias primas empleadas en cada caso. Al observar el peso para cada sector se observa que éste tiende a ser mayor en promedio en el sector oeste (media=468 g, desvío=187 g) que en el norte (media=185 g, desvío=144 g).

Las muestras del sector norte poseen una media de volumen de $142 \mathrm{~cm}^{3}$, mientras que en el oeste es de 207 $\mathrm{cm}^{3}$. Sin embargo se observa una mayor dispersión de valores para el primero, con un desvío estándar de 118 $\mathrm{cm}^{3}$, mientras que el desvío para el sector oeste es de 43 $\mathrm{cm}^{3}$. El promedio de peso en la costa norte es de $185 \mathrm{~g}$ con un rango que oscila entre los 6 y $459 \mathrm{~g}$, en la costa oeste en cambio, el promedio es mayor (468 g) así como el rango que va de 28 a $668 \mathrm{~g}$. Este patrón se repite para el peso específico (indicando que las diferencias observadas no son sólo diferencias de tamaño) ya que éste es menor en el sector norte (media $1.29 \mathrm{~g}$, rango de 1.02 a 1.57 g) que en el sector oeste con un promedio de $2.24 \mathrm{~g}$ y un rango de 1.43 a $3 \mathrm{~g}$. Estas diferencias de peso y peso específico parecen vincularse a la mayor frecuencia en la costa oeste de rocas hematíticas con contenido de hierro provenientes del Manto Ferrífero que están localmente disponibles (Alberti 2015), mientras que una mayor frecuencia de rocas carbonáticas (calizas), de escaso peso específico, ha sido empleada en el sector norte. El test de la t para medias señala que ambas muestras poseen diferencias significativas en peso $(t=4.07, p<0.001)$. En el caso del volumen el test de la t para varianzas desiguales y el de permutación no señalan diferencias significativas, lo que se debe a la amplia dispersión de volúmenes que muestra el sector norte $(\mathrm{t}=1.48, \mathrm{p}>0.05$, permutación $p>0.05)$. En cuanto al peso específico, los resultados son acordes a lo observado en los gráficos de caja con diferencias significativas para ambas muestras (t varianzas desiguales, $t=6.68 p<0.001$, permutación $p<0.001$ ).

\section{Análisis morfológico}

Se utilizaron 12 harmónicas ya que se observó que visualmente ese número de parámetros describía correctamente la morfología general de estos artefactos 

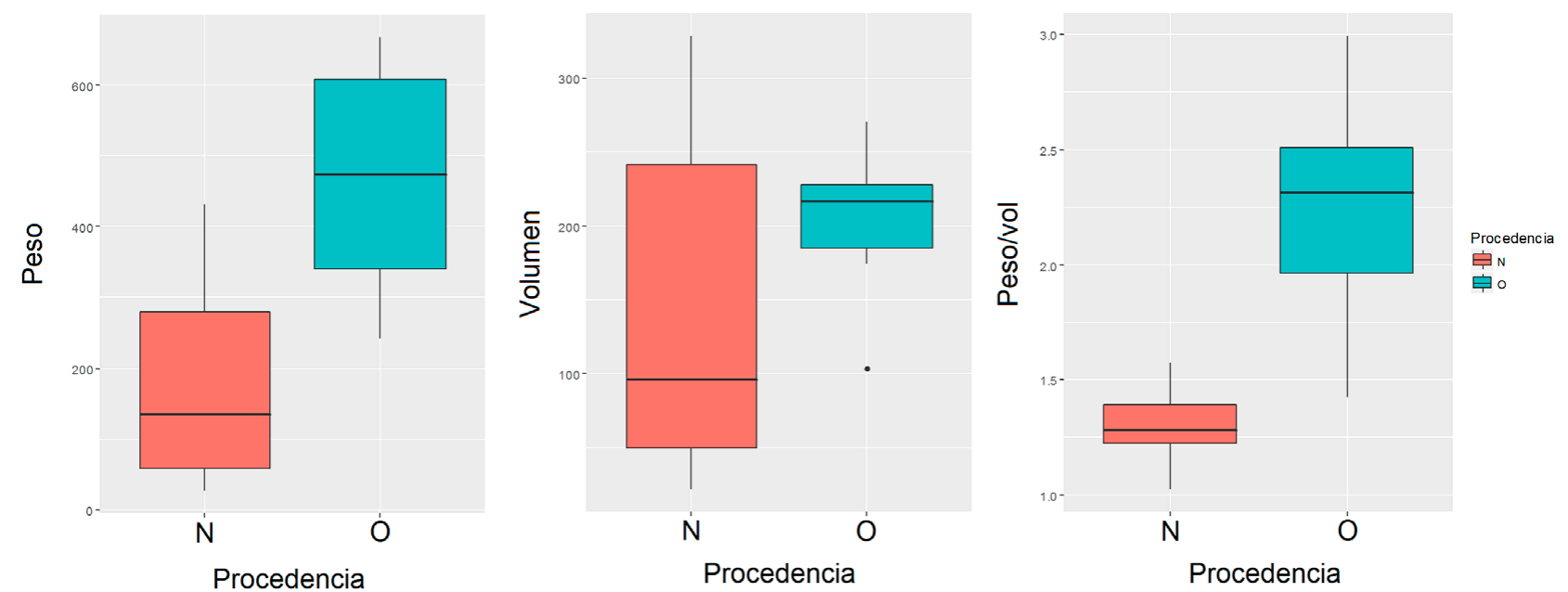

Figura 3. Gráfico de caja de las variables métricas por sector.

Figure 3. Metric variables box plot by area.

(Figura 4). Estos coeficientes fueron posteriormente empleados para realizar el análisis de componentes principales para cada una de las vistas (Figura 5 y 6).

Estos componentes capturan las tendencias generales de variación morfológica para ambas vistas. En el caso de la vista frontal (Figura 5) los dos primeros componentes capturan cerca del $83 \%$ de la variación total en el contorno. El primer componente, que explica un 64\% de la variación, muestra la tendencia de morfologías circulares levemente romboidales (valores negativos PC1) a morfologías con ambos polos comprimidos (valores positivos PC1). El segundo componente explica aproximadamente un 19\% y muestra la tendencia entre formas más cuadrangulares (valores negativos) a romboidales (valores positivos). En general, pocos casos se distribuyen de forma pronunciada sobre este segundo eje.

El primer componente de la vista lateral explica un $85 \%$ de la variación, mientras que el segundo poco más del $6 \%$. En el primer caso se observa que este componente muestra una tendencia asimétrica en la deformación, donde uno solo de los polos se comprime y tiende a hundirse (valores negativos) partiendo de formas circulares (valores positivos). El segundo componente también muestra una relación asimétrica con una tendencia a formas más angulosas y romboidales (valores negativos) a con uno de los polos hundido, aunque de una forma muy localizada (valores positivo). La proporción de la varianza explicada por este último componente así como la extensión de

\section{Frontal}

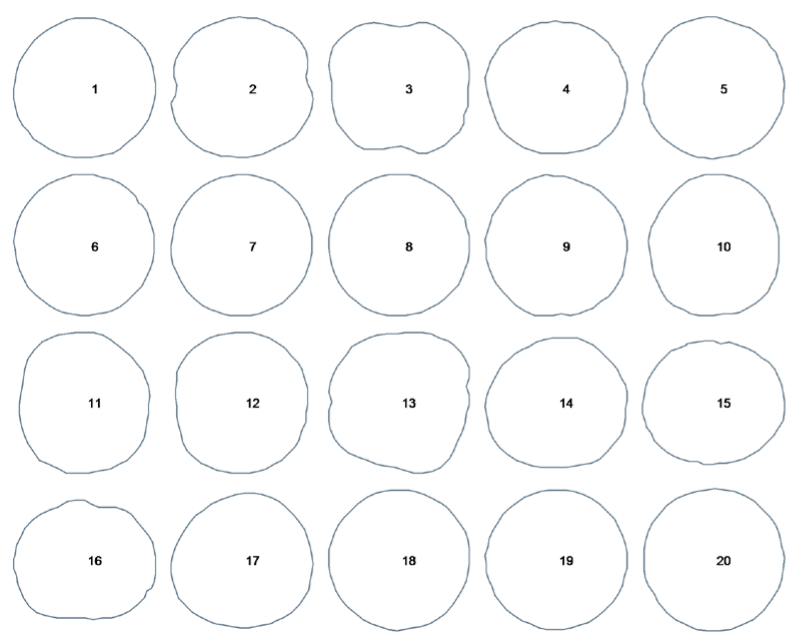

Lateral

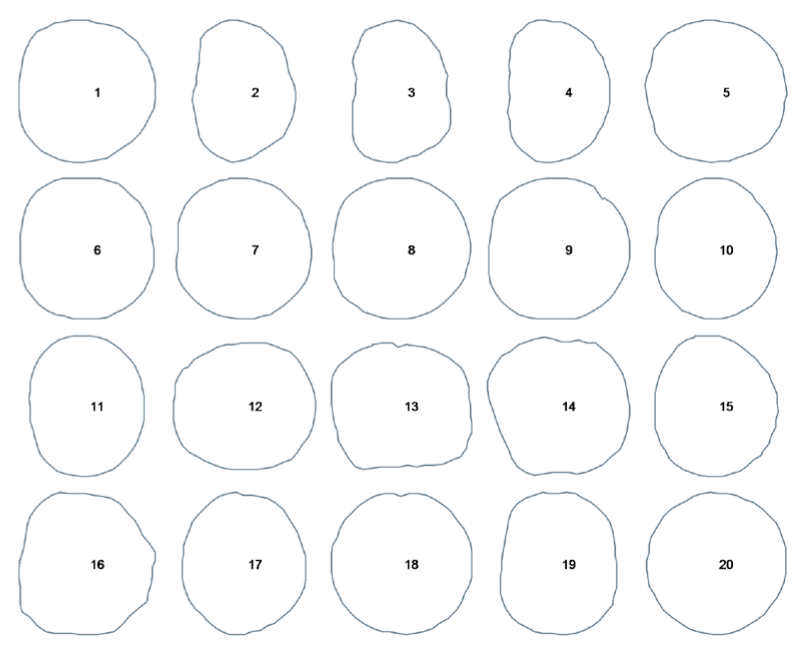

Figura 4. Conjunto de contornos obtenidos mediante el análisis de Fourier elíptico para las vistas frontal y lateral.

Figure 4. Set of outlines obtained by means of elliptic Fourier analysis to the frontal and lateral views. 


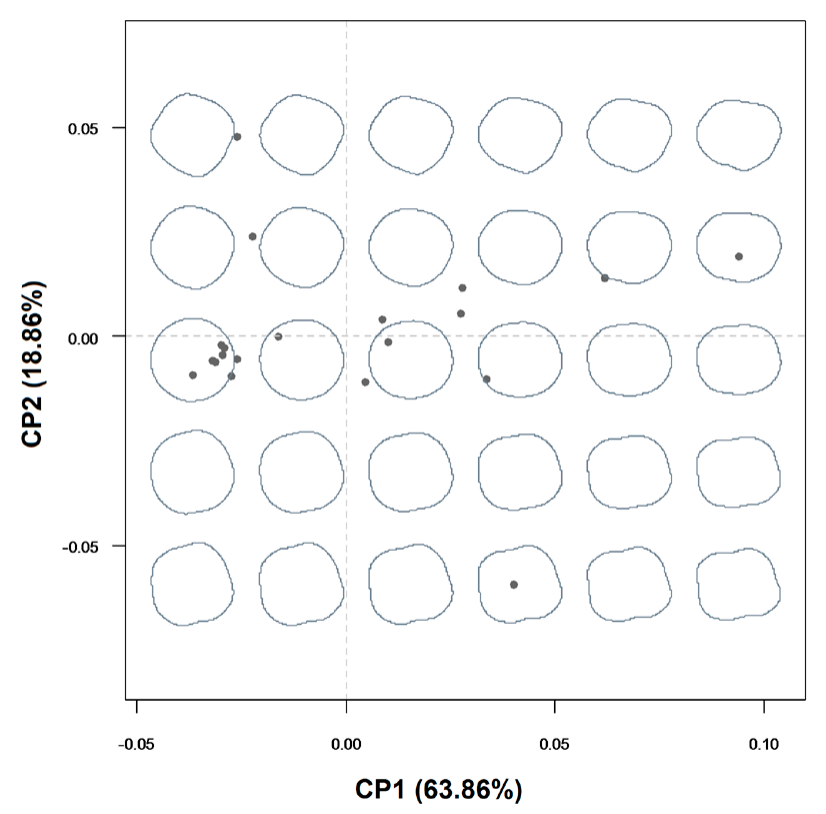

Figura 5. Dos primeros componentes principales de forma de la vista frontal. Los contornos representan la deformación esperada para ese sector del espacio morfológico a partir de la forma promedio.

Figure 5. Two first shape main components of the frontal view. The outlines represent the expected deformation for that portion of the morphological space from the average form.

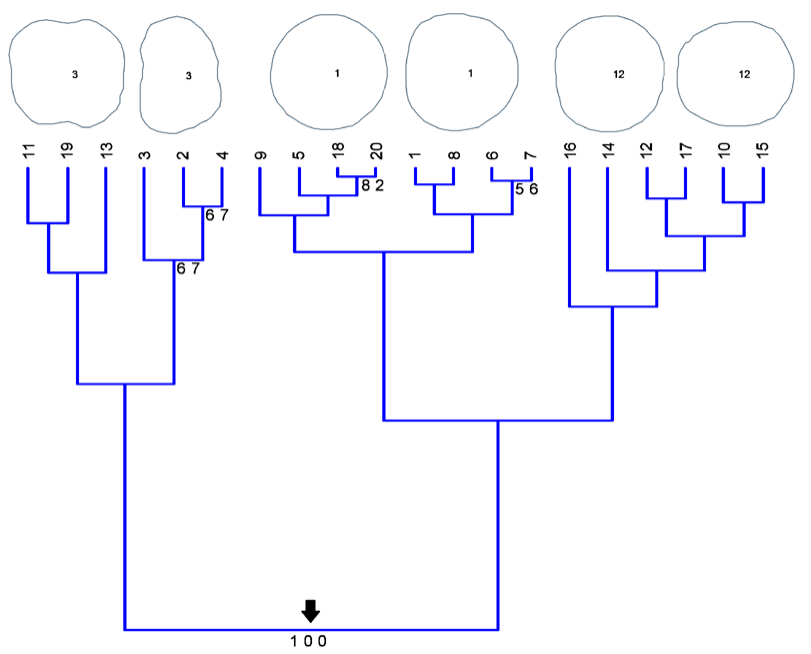

Figura 7. Agrupamiento resultante de la aplicación del método de Ward. Se señalan aquéllos agrupamientos soportados por más del $50 \%$ del procedimiento de remuestreo. Los contornos muestran la tendencia de deformación en cada uno de los grupos, representada por uno de los casos que lo integran.

Figure 7. Grouping resulting from the application of the Ward method. Those groupings supported by more than $50 \%$ of resampling procedure are listed. The outlines show the trend of deformation in each of the groups, represented by one of the cases in each one.

los casos sobre el espacio morfológico, sugiere que este fenómeno está relativamente restringido a pocos artefactos.

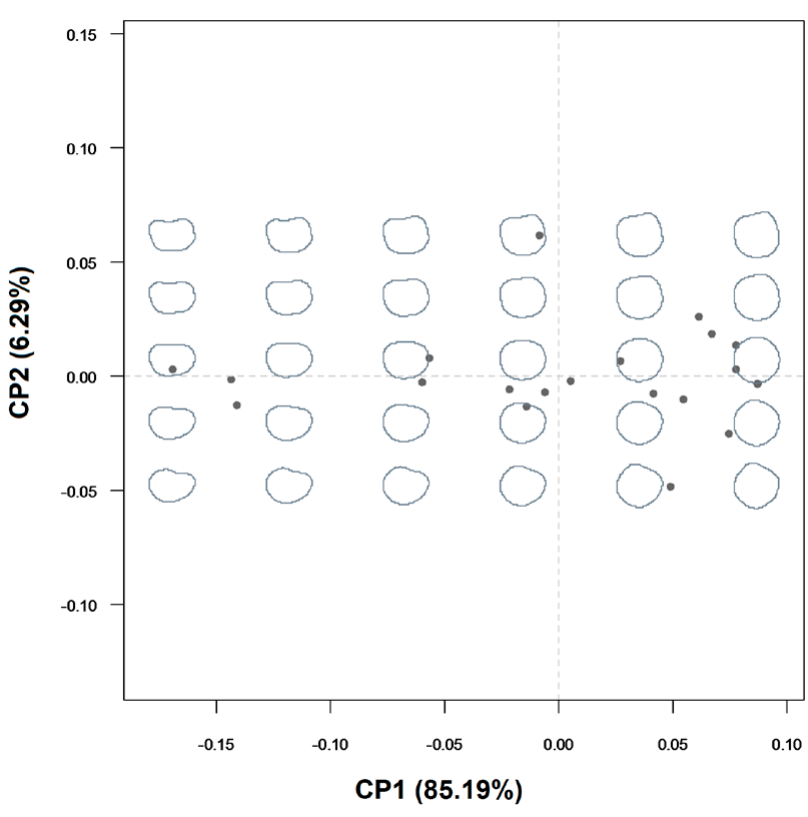

Figura 6. Dos primeros componentes principales de la vista lateral. Los contornos representan la deformación esperada para ese sector del espacio morfológico a partir de la forma promedio.

Figure 6. Two first shape main components of the lateral view. The outlines represent the expected deformation for that portion of the morphological space from the average form.

Con el fin de estudiar la existencia de grupos morfológicos dentro de la variación estudiada, se tomaron los tres primeros componentes principales de ambas vistas (que explican alrededor del $90 \%$ del total de la variación morfológica en ambos casos) y se realizó un análisis de agrupamiento utilizando el método de Ward (ver Metodología). Los resultados (Figura 7), muestran el ordenamiento de la variación en dos grandes grupos soportados por un $100 \%$ de interacciones del remuestreo y que muestran una alta disimilitud entre sí. El subconjunto de mayor tamaño está conformado por los casos de circularidad perfecta o aquéllos que poseen formas ligeramente alargadas $u$ ovales en alguna de las vistas. El segundo grupo, más pequeño, agrupa los casos de morfología cuadrangular y de perfil relativamente achatado.

A niveles de disimilitud menos importantes, hay pocos agrupamientos robustos (en términos del soporte de remuestreo) que indican principalmente la existencia de pares de casos muy similares. Estas diferencias morfológicas no se vinculan a la localización de las muestras, ya que el test discriminante para estos seis componentes divididos por pertenencia no muestra valores significativos (Hotelling $t^{2}=8, p>0.05$, porcentaje correctamente clasificado 75\%). Esto sugiere que otros factores, como tamaño o materias primas pueden ser factores más explicativos.

Con el fin de determinar qué otros factores pueden haber 


\begin{tabular}{cccccccccc}
\hline & CPFront1 & CPFront2 & CPFront3 & CPLat1 & CPLat2 & CPLat3 & Volumen & Peso & Peso/Vol \\
\hline CPFront1 & & - & - & - & - & - & $p<0.01$ & - & - \\
CPFront2 & 0 & & - & - & - & - & $p<0.05$ & - & $p<0.01$ \\
CPFront3 & 0 & 0 & & - & $p<0.01$ & - & - & - & - \\
CPLat1 & 0.31 & 0.19 & -0.15 & & - & - & - & - & $p>0.05$ \\
CPLat2 & -0.18 & -0.29 & -0.67 & 0 & & - & - & - & - \\
CPLat3 & -0.23 & -0.19 & 0.22 & 0 & 0 & & - & - & - \\
Volumen & $0.60^{*}$ & $-0.44^{*}$ & 0.13 & 0.09 & 0.06 & 0.27 & & $p<0.01$ & - \\
Peso & $0.51^{*}$ & $-0.58^{*}$ & 0.21 & 0.11 & 0 & 0.17 & 0.81 & 0.01 & $p<0.01$ \\
Peso/Vol & 0.19 & $-0.59^{*}$ & 0.26 & -0.01 & -0.09 & 0.05 & 0.34 & 0.80 & \\
\hline
\end{tabular}

Tabla 2. Valores de correlación (triángulo inferior) y de probabilidad significativos (triángulo superior), entre las variables métricas y morfológicas. ${ }^{*}$ Correlaciones significativas entre forma y tamaño.

Table 2. Correlation values (lower triangle) and significant probability (upper triangle) between the metric and morphological variables. *Significant correlations between shape and size.

jugado un papel relevante, un tercer paso fue explorar las relaciones entre forma (representada por los tres componentes de cada una de las vistas) y tamaño (ver Metodología). Los resultados del análisis de correlación lineal entre estas variables señalan que morfología y tamaño están relacionados en la vista frontal y en lo que respecta a los dos primeros componentes (Tabla 2):

La tendencia de la correlación señala que formas de mayor peso y volumen tienden a poseer contornos relativamente más ovalados o alargados, mientras que las más pequeñas o de menor peso y volumen contornos relativamente más irregulares y angulosos. También se observa una correlación media y significativa entre peso específico y el segundo componente principal de la vista frontal, la cual sugiere que aquellas formas de mayor peso específico tienden a poseer contornos más angulosos. En general, el patrón de vinculación entre forma y tamaño parece estar vinculado en parte a aspectos del diseño independientes a las materias primas empleadas.

Los análisis de los datos cualitativos arrojaron resultados dispares. Por un lado se comprobó que las diferencias detectadas en la cantidad de ejemplares enteros y fragmentados $\left(X^{2}=4.51, p=0.10, n=42\right)$, los tipos de acabado en relación con las bolas con y sin surco $\left(X^{2}=0.41, p=0.81, n=42\right)$, en la cantidad de ejemplares con diferentes tipos de acabado entre costas $\left(X^{2}=0.46\right.$, $\mathrm{p}=0.79, \mathrm{n}=42$ ) y las diferencias entre bolas con y sin surco en la costa oeste $\left(X^{2}=0.24, p=0.88, n=18\right)$, no resultaron estadísticamente significativas, con lo que se deberían al azar. En cambio, resultaron significativas diferencias como la cantidad de bolas con y sin surco entre costas $\left(X^{2}=7.41\right.$, $\mathrm{p}<0.01, \mathrm{n}=42, \mathrm{P}=0.89)$, los tipos de materias primas que se usaron en cada costa $\left(X^{2}=27.12, p<0.01\right.$. $\left.n=41, P=0.77\right)$, la cantidad de bolas con y sin surco por materia prima para la costa norte $\left(X^{2}=5.25, p<0.05 . n=23, P=0.56\right), y$ los tipos de acabado por materia prima en cada una de las costas $\left(X^{2}=10, p<0.05, n=23, P=0.45\right.$ para el caso de la costa norte, $y X^{2}=10.44, p<0.05, n=18, P=0.35$ para el caso de la oeste). En este sentido, la cantidad de bolas con y sin surco y las materias primas utilizadas pueden estar vinculadas entre sí, teniendo en cuenta que la potencia de este test ha sido baja. En la costa norte la presencia de bolas con surco es efectivamente mayor que en la oeste, en particular en las rocas sedimentarias (calizas) que son más blandas que los otros tipos de materia prima utilizados y cuya presencia mayoritaria en esta costa se debería a factores no relacionados con el azar. Se destaca al respecto que estas dos variables han sido las que poseen una diferencia más robusta entre sí en relación al valor del test y su potencia. En la costa oeste, en cambio, no existen diferencias estadísticamente significativas en la cantidad de bolas con y sin surco manufacturadas sobre diferentes rocas pero cabe destacar que en allí la principal roca utilizada para la manufactura de bolas fue la hematita que posee una dureza muy alta y, por lo tanto, es más difícil de formatizar que las rocas sedimentarias más blandas. Finalmente, en relación con el acabado que presentan estos artefactos, el acabado 1 es mayoritario en ambas costas pero mientras que en la costa norte se presenta principalmente en las rocas sedimentarias, lo que podría responder a la acción de agentes tafonómicos que darían como resultado la abrasión de estas rocas (ver más adelante), en la costa oeste aparece sobre las bolas manufacturadas en hematita, lo cual indica una clara intención y habría implicado un mayor esfuerzo e inversión energética para lograrlo.

\section{Discusión y conclusiones}

El alto porcentaje de la muestra aquí estudiada de artefactos que se presentan enteros (50\% del total) nos ha permitido realizar análisis de forma que posibilitan la diferenciación de dos grandes grupos morfológicos: uno de formas de tamaño en general mediano-pequeño de 
contorno irregular aunque tendiente a cuadrangulares o rectangulares, de vista lateral más bien plana y achatada, y otro de formas más esféricas con vista lateral esférica o sub-oval, de tamaño en general mayor (ver acápite anterior). Esto está sustentado, además, por las correlaciones entre forma y peso, volumen y peso/ volumen. Asimismo cada sector posee diferencias no en forma pero sí en tamaño, ya que en promedio el sector oeste posee artefactos de un mayor peso y peso específico, mientras que en el otro sector el promedio para estas variables es significativamente más bajo. El amplio rango de dispersión que poseen estos valores podría deberse a diferentes funcionalidades de estos artefactos (bolas más grandes y pesadas vs. bolas más pequeñas y livianas). De observarse una tendencia (como podría inferirse del análisis discriminante que separa un $75 \%$ de los casos correctamente aunque de forma no significativa), ésta se relacionaría con la distribución desigual de algunas materias primas y a las rocas que fueron empleadas en cada caso. Las rocas sedimentarias son de origen local en la costa norte del golfo y aparecen en fuentes secundarias, ubicuas y abundantes en el ambiente (Alberti 2012, 2015; Favier Dubois y Alberti 2014). En cambio, la hematita es local en la costa oeste del golfo y habría sido obtenida del Manto Ferrífero, formación geológica de amplia distribución en la costa oeste con afloramientos puntuales en ciertos sectores del espacio (Alberti y Cardillo 2015; Alberti 2015). Finalmente, las rocas volcánicas aparecen en proporciones mayores en las fuentes secundarias de la costa norte, pero también se encuentran en el sector oeste del golfo. Todo esto apunta a un uso predominantemente local para la manufactura de las bolas, independientemente de su forma y tamaño.

La existencia de surco en las bolas tiene que ver con la forma de agarre del arma: a aquéllas que lo poseen se les habría amarrado un tiento alrededor, mientras que las que no se habrían usado retobadas (Martinic 1995; Vecchi 2010; entre otros). La presencia de surco en rocas duras como la hematita indicaría una clara intencionalidad de conservación del instrumento debido a la alta inversión de energía que significaría la elaboración del surco en dichas bolas. En este sentido, la presencia de bolas con surco es más alta en la costa norte, en particular en los artefactos más pequeños manufacturados sobre calizas. Estas pequeñas esferas no habrían sido utilizadas para la caza de animales grandes (ver más adelante) debido, justamente, a su bajo peso específico y su tamaño pequeño. En la bibliografía hemos relevado casos de caza de animales de porte menor, como aves y mulitas (Musters 1964) mediante el uso de boleadoras, pero esta es una hipótesis que, por el momento, no puede ser contrastada (ver supra).

Respecto de las marcas de formatización, resulta relativamente bajo el porcentaje total de la muestra que no las posee (31\%) lo que indicaría que, a pesar del esfuerzo invertido en la manufactura de los instrumentos y en su acabado final, no habría existido una intención de lograr esferas perfectas sin ningún tipo de marca, lo cual habría disminuido en cierto punto la energía invertida para la fabricación de los artefactos. Esto se relaciona, además, con el alto porcentaje de la muestra que presenta acabados de grado 1 o 0 .

En relación con lo anterior, el grado 1 de acabado que muestra la mayoría de estos artefactos podría deberse a la acción de procesos tafonómicos en el caso de los instrumentos provenientes de la costa norte, debido a la baja dureza de las rocas sedimentarias que pueden abradirse fácilmente por la acción de los agentes tafonómicos actuantes en el área (Carranza 2015). En este sentido, estudios tendientes a la observación y control de estos fenómenos están siendo llevados adelante en el área de estudio. En el caso de las rocas con alto contenido de hierro, la presencia de pulido y brillo podría indicar dos procesos distintos: o bien estas bolas tienen una antigüedad mayor, con lo que los procesos tafonómicos serían los causantes de la presencia de este acabado gracias a su acción durante un tiempo más prolongado (necesario para causar algún daño en estas rocas más duras) (Alberti y Carranza 2014; Carranza 2015), o bien la presencia de este acabado es intencional, lo cual apoyaría también la intención de conservación de estos instrumentos, que fueron hallados enteros en su mayoría. Cabe destacar que la mayoría de las bolas recuperadas en la costa oeste fueron halladas en el sector del Cañadón del Puma, frente al Islote Lobos. Allí fue encontrada incluso una concentración de tres de estos artefactos, juntos y en perfectas condiciones (Borella et al. 2015), lo que podría responder a un almacenamiento para uso futuro o un escondrijo. Las investigaciones en este sector de la costa oeste han comenzado a ser intensificadas recientemente, con lo que, a futuro, con la obtención de fechados y el análisis del registro faunístico esta afirmación podrá verse sustentada o será descartada.

Debido a su bajo peso específico, las bolas manufacturadas sobre rocas sedimentarias no habrían tenido la misma función que aquéllas fabricadas en rocas más duras y más pesadas (ver supra). Ya ha sido planteado el uso de estos esferoides más pequeños y livianos como posibles pesas de línea (Scartascini 2014); su virtual ausencia en la costa oeste del golfo respondería al empleo de otras técnicas de captura de peces que habrían sido empleadas en esa zona (Scartascini 2014). En este sentido, las bolas más pequeñas, de contornos más irregulares, en caso de haber sido usadas de esta forma, no habrían necesitado de una alta inversión de energía para su manufactura ya que no habría sido necesario que poseyeran requisitos aerodinámicos para el vuelo, como en el caso de los artefactos más grandes que podrían haber sido usados para la caza de animales mayores a través de la combinación de varios ramales (ver supra). Son los artefactos de tamaños mayores los que presentan las formas más ovaladas y de contornos más regulares. Este 
tipo de forma, en bolas hechas sobre rocas más duras, habría requerido una mayor inversión de energía para su formatización con lo que quizás habrían sido artefactos más conservados.

A partir de este trabajo fue posible delinear tendencias generales en la muestra de bolas líticas recuperadas en la costa del golfo San Matías. La ampliación de la muestra, en conjunto con los análisis zooarqueológicos permitirá, a futuro, afinar nuestro conocimiento sobre el uso de esta tecnología en la costa de Norpatagonia.

Buenos Aires, 14 de abril 2016

\section{Agradecimientos}

Este trabajo fue financiado por el proyecto PIP CONICET 112-201101-00589, dirigido por los Dres. F. Borella y M. Cardillo, y el proyecto PICT ANPCyT, dirigido por el Dr. C. Favier Dubois. A los Dres. Rodrigo Vecchi, Karen Borrazzo, Florencia Borella y Luis Borrero por atender nuestras consultas y proporcionarnos bibliografía de gran utilidad para la escritura de este trabajo. A los evaluadores anónimos cuyas sugerencias ayudaron a mejorar nuestro trabajo.

\section{Bibliografía}

Alberti, Jimena. (2012). Fuentes de rocas y uso de materias primas líticas en Bahía Final 6, costa norte del golfo San Matías (Río Negro, Argentina). Intersecciones en antropología, 13(1), 227-249.

Alberti, J. (2015). Disponibilidad y explotación de fuentes de materias primas líticas durante el Holoceno medio y tardío en la costa del golfo San Matías, Río Negro, Argentina. Un enfoque regional. Universidad de Buenos Aires. Tesis Doctoral Inédita. 324 pp.

Alberti, Jimena, \& Carranza, Eugenia. (2014). Primera caracterización de los conjuntos líticos provenientes de depósitos de tipo conchero en la costa del golfo San Matías (Río Negro, Argentina). La zaranda de ideas, 11, 47-64.

Alberti, J., \& Cardillo, M. (2015). Primary and secondary lithic raw material sources along the western coast of San Matías Gulf (Río Negro province, Argentina): A first approach to their spatial variability. Quaternary International, 373, 63-71. JOUR. http://doi.org/http:// dx.doi.org/10.1016/j.quaint.2014.09.06

Alberti, J., \& Fernández, V. (2015). Propuesta clasificatoria para las materias primas líticas en Patagonia (Argentina). Arqueología, 21(2), 211-235.

Alberti, J., Cardillo, M., \& Favier Dubois, C. M. (2015). Fuentes de materias primas líticas en la costa del golfo San Matías (provincia de Río Negro, Argentina): Una síntesis regional. Intersecciones en antropología, 16(Supl. 1), 27-37.

Babot, P. (2004). Tecnología y utilización de artefactos de molienda en el noroeste prehispánico. Facultad de Ciencias Naturales e I. M. L., Universidad Nacional de Tucumán. Tesis Doctoral Inédita. Facultad de Ciencias Naturales e I. M. L., Universidad Nacional de Tucumán.

Bonhomme, V., Picq, S., Gaucherel, C., \& Claude, J. (2014). Momocs: Outline Analysis Using R. Journal of Statistical Software, 56(13), 1 - 24. doi:http://dx.doi.org/10.18637/ jss.v056.i13

Bird, J. B. (1993). Viajes y arqueología en Chile austral. Punta Arenas, Magallanes, Chile: Ediciones de la Universidad de Magallanes.

Borella, F. (2006). ¿Dónde están los lobos en la costa norpatagónica? Explorando vías para resolver el registro arqueofaunístico. Werken, 9, 97-114.

Borella, F., Cardillo, M., Favier Dubois, C., \& Alberti, J. (2015). Nuevas investigaciones arqueológicas entre Punta Pórfido y Punta Odriozola: implicancias para el entendimiento de la dinámica de las ocupaciones humanas en la costa oeste del golfo San Matías (Río Negro). Relaciones de la Sociedad Argentina de Antropología, 40(1), 233-252.

Borrazzo, K., \& Etchichury, M. (2013). Estudio de las materias primas líticas utilizadas para la manufactura de bolas en el norte de Tierra del Fuego (Argentina). Arqueología, 19(2), 305-324.

Carranza, E. (2015). "Análisis de conjuntos líticos de superficie desde una perspectiva tafonómica. Estudios en la costa norte del golfo San Matías, Río Negro, Argentina." Facultad de Filosofía y Letras, Universidad de Buenos Aires, 114 páginas. Tesis de Licenciatura Inédita. Facultad de Filosofía y Letras, Universidad de Buenos Aires.

Cohen, J. (1988). Statistical power analysis for the behavioral sciences. Segunda Edición. Elsevier, Amsterdam.

Davis, J. C. (1986). Statistics and data analysis in geology. New York: Wiley.

Favier Dubois, C. \& Alberti, J. (2014). Materias primas líticas en la costa norte del golfo San Matías (Río Negro, Argentina): distribución de fuentes y tendencias generales en su aprovechamiento. Revista del Museo de Antropología, 7(1), 93-104.

Favier Doubois, C., \& Borella, F. (2011). Contrastes en la costa del golfo: una aproximación al estudio del uso 
humano del litoral rionegrino. En F. Borella \& M. Cardillo (Eds.), Arqueología de Pescadores y Marisqueadores en Nordpatagonia. Descifrando un Registro de más de 6.000 Años (pp. 13-42). Buenos Aires: Dunken.

Favier Dubois, C., Borella, F., Manzi, L., Cardillo, M., Lanzellotti, S., Scartascini, F., Carolina, M. \& Borges Vaz, E. (2008). Aproximación regional al registro arqueológico de la costa rionegrina. En I. Cruz \& S. Caracotche (Eds.), Arqueología de la costa patagónica. Perspectivas para la conservación (pp. 50-68). Río Gallegos.

Gero, J., \& Mazzullo, J. (1984). Analysis of artifact shape using Fourier series in closed form. Journal of Field Archaeology, 11, 315-322.

González, A. R. (1954). La boleadora: sus áreas de dispersión y tipos. Revista del Museo de la Universidad Eva Perón (Nueva Serie), 4, 133-292.

González Díaz, E.., \& Malagnino, E.. (1984). Geomorfología de la provincia de Río Negro. Actas del IX Congreso Geológico Argentino, 1-159, Secretaría de Minería, Buenos Aires.

Hammer, Ø., Harper, D. \& Ryan, P.. 2001. PAST. Palaeontological Statistics software package for education and data analysis. Palaeontologia Electronica, 4(1), 1-9.

Hope, A. (1968). A simplified Monte Carlo significance test procedures. Journal of the Royal Statistical Society. Series B (Methodological), 30(3), 582-598.

Husson, F., Josse, J. \& Pagès, J. (2010). Principal component methods - hierarchical clustering - partitional clustering: why would we need to choose for visualizing data? Technical report online. http://factominer.free.fr/ docs/HCPC_husson_josse.pdf (última consulta: febrero 2016).

Kaufman, L. \& Rousseeuw, P. (1990). Finding groups in data. An introduction to cluster analysis. John Wiley \& Sons, New York.

Kuhl, F., \& Giardina, C. (1982). Elliptic Fourier features of a closed contour. Computer Graphics and Image Processing, 18, 236:258.

Legendre, P., \& Legendre L.. (2012). Numerical Ecology. Tercera Edición. Elsevier, Ámsterdam.

Mansur-Franchomme, M., Orquera L.. A. y Piana, E. L. (1987-1988). El alisamiento de la piedra entre los 1988 cazadores-recolectores: El caso de Tierra del Fuego. Runa, XVII-XVIII, 111-205.

Martinic, M. (1995) Los Aonikenk. Historia y Cultura. Punta Arenas: Ediciones Universidad de Magallanes.

Moreno, E.. (2008). Arqueología y etnohistoria de la Costa
Patagónica Central en el Holoceno Tardío. Rawson: Fondo Editorial Provincial, Secretaría de Cultura de Chubut.

Moreno, E.., Castro, A., \& Pepe, F.. (2000). El rompecráneo: un artefacto probablemente destinado a la caza de pinnípedos en la costa patagónica. Desde el país de los gigantes. Perspectivas arqueológicas en Patagonia, Tomo II, 563-571, Río Gallegos: Universidad Nacional de la Patagonia Austral.

Musters, G. ([1869-1870] 1964). Vida entre los patagones. Un año de excursiones por tierras no frecuentadas, desde el Estrecho de Magallanes hasta el Río Negro. Buenos Aires: Ediciones Solar/Hachette.

Oswalt, W. (1976). An anthropological analysis of foodgetting technology. Nueva York: John Wiley \& Sons.

R Development Core Team (2009). R: A language and environment for statistical computing. Vienna, Austria: $R$ Foundation for Statistical Computing. http://www.Rproject.org (último acceso: febrero 2016).

Rohlf, F. (2006). TPS serie softwares. http//life. bio.sunysb. edu/ Corp (último acceso: febrero 2016).

Scartascini, F. (2014). "Arqueología de la pesca en la costa rionegrina, Patagonia Argentina." Facultad de Filosofía y Letras, Universidad de Buenos Aires, 313 páginas. Tesis Doctoral Inédita. Facultad de Filosofía y Letras, Universidad de Buenos Aires.

Torres Elgueta, J. (2009). Bolas líticas y sus procesos de manufactura, en contextos de cazadores recolectores terrestres del norte de Tierra del Fuego. Evidencias desde el Holoceno Medio hasta 1500 años AP. En M. Salemme, F. Santiago, M. Álvarez, E. Piana, M. Vázquez, \& M. Mansur (Eds.), Arqueología de Patagonia: Una mirada desde el último confín (pp. 393-411). Ushuaia: Editorial Utopías.

Torres Elgueta., J., \& Morello, F. (2011). Bolas, manijas y guijarros piqueteados de la laguna Thomas Gould Patagonia meridional, XII región de Magallanes. En L. A. Borrero \& K. Borrazzo (Eds.), Bosques, montañas y cazadores: investigaciones arqueológicas en Patagonia Meridional (pp. 211-239). Buenos Aires: CONICETIMHICIHU.

Vecchi, R.. (2007). Cazando en la frontera: El uso de la boleadora en la región pampeana bonaerense durante el siglo XIX". Undécimo Congreso de Historia de Los Pueblos de la provincia de Buenos Aires, Edición digital en CD-ROM. Archivo Histórico de la Provincia de Buenos Aires Dr. Ricardo Levene, La Plata.

Vecchi, R.. (2010). "Bolas de boleadora en los grupos de cazadores-recolectores de la Pampa bonaerense." Facultad de Filosofía y Letras, Universidad de Buenos Aires, 364 páginas. Tesis Doctoral Inédita. Facultad de Filosofía y Letras, Universidad de Buenos Aires. 